\title{
Associations between C-reactive protein and white blood cell count, occurrence of delayed cerebral ischemia and poor outcome following aneurysmal subarachnoid hemorrhage: a systematic review and meta-analysis
}

\author{
Xinlong Ma ${ }^{1} \cdot$ Feng Lan $^{2} \cdot$ Yuqi Zhang ${ }^{1}$
}

Received: 20 August 2020 / Accepted: 7 September 2020 / Published online: 9 January 2021

(c) Belgian Neurological Society 2021

\begin{abstract}
This review and meta-analysis investigated associations of systemic inflammatory marker C-reactive protein (CRP) and white blood cell count (WBC) with occurrence of delayed cerebral ischemia (DCI) and poor functional outcome after aneurysmal subarachnoid hemorrhage (aSAH). Pubmed, EMBASE, and CENTRAL databases were searched until November 30, 2019, selecting prospective and retrospective studies of patients with spontaneous SAH due to ruptured aneurysm. Outcome measures were occurrence of DCI, defined as new focal neurological deficit or a deterioration of consciousness; and/or a new infarct on computed tomography or magnetic resonance imaging that was not visible initially. Occurrence of poor functional outcome at follow-up were measured by modified Rankin Scale or Glasgow outcomes scale. Fifteen studies analyzing data of 3268 patients with aSAH were included. Meta-analysis revealed early increase in CRP was significantly associated with higher risk of occurrence of DCI (pooled OR 1.30, 95\% CI 1.10-1.54; $P=0.002$ ), whereas not with poor functional outcome (pooled OR 1.02, 95\% CI 1.00-1.04, $P=0.052$ ). No significant associations between early increase in WBC and DCI (pooled OR $1.13,95 \%$ CI $0.95-1.34 ; P=0.179)$ were observed, whereas increase in WBC was significantly associated with increased risk of poor functional outcome (pooled OR 1.17, 95\% CI 1.07-1.28, $P=0.001$ ). Early increase in blood CRP appears to correlate with DCI after SAH, while increase in WBC correlates with poor functional outcome. However, strong conclusion cannot be made due to the small study number, between-study heterogeneity and suspicion of uncontrolled factors. Whether early phase CRP and WBC may serve as prognostic markers for aSAH needs more investigation.
\end{abstract}

Keywords Aneurysmal subarachnoid hemorrhage $\cdot$ Biomarker $\cdot$ C-reactive protein $\cdot$ Delayed cerebral ischemia $\cdot$ White blood cell count

Xinlong Ma and Feng Lan contributed equally to the work.

Electronic supplementary material The online version of this article (https://doi.org/10.1007/s13760-020-01496-y) contains supplementary material, which is available to authorized users.

Yuqi Zhang

Yuqi9597@sina.com

1 Department of Neurosurgery, Yuquan Hospital, Tsinghua University, No. 5 Shijingshan Road, Shijingshan District, Beijing 100049, China

2 Pediatric Intensive Care Unit, The First Affiliated Hospital of Xinjiang Medical University, Ürümqi, China

\section{Introduction}

Subarachnoid hemorrhage (SAH) is a prominent cause of morbidity and mortality worldwide, with overall incidence reported to be about 9 per 100,000 person-years [1] and mortality estimated to be about $35 \%$ [2]. In about $85 \%$ of cases, the underlying cause is ruptured intracranial aneurysm, which is associated with poorer outcomes than non-aneurysmal cases (perimesencephalic and various other types) [3]. Development of aSAH is shown to peak in middle-aged and older adults, particularly between the ages of 50 and 60 years, and is $1-6$ times more common among older adult women than men [1].

Although clinical decline after aSAH may arise from etiologies such as hydrocephalus, rebleeding, metabolic 
disturbances or infection [4], delayed cerebral ischemia (DCI) is a frequent cause of poor neurological outcomes and death in patients who survive the initial bleeding of aSAH [5]. DCI also may progress to cerebral infarction, resulting in severe disability or death in about $50 \%$ of SAH patients, and is deemed the most important adverse prognostic factor for poor outcome of aSAH [6]. Patients who develop DCI, may manifest unifocal or multifocal areas of ischemia, typically between days 4 and 14 after the initial hemorrhage, and not restricted to the arterial supply or borderzone areas [4]. Although vasospasm figures prominently in DCI development, the pathogenesis is believed to be multifactorial [7, 8].

The currently accepted predictive factors for long-term neurologic outcomes after SAH, especially DCI and poor functional outcomes, include age and clinical severity [9, 10]. A review study investigating hemostatic or fibrinolytic parameters that may predict DCI also reported that levels of von Willebrand factor and platelet activating factor were higher in SAH patients with DCI than in those without DCI [4]. Recent evidence of serum parameters that serve as biomarkers for predicting SAH outcomes suggests that various inflammatory, fibrinolytic and hematologic factors may be associated with poor outcomes after SAH, including elevated glucose level [11], low platelet count [12], elevated CRP [13-15], and white blood cell (WBC) count [15, 16].

Having a biomarker which is convenient to obtain in assessing patients' individual risk for poor outcome after aSAH would have tremendous clinical value. Among the potential biomarkers, CRP and WBC are markers of systemic inflammation which are routinely collected on admission have drawn much attention of research. However, the relationships between these parameters at early phase of admission and the occurrence of DCI and poor outcome at follow-up remains ambiguous, and no meta-analysis has yet estimated them. To investigate whether these parameters may aid in identifying patients at high risk of poor prognosis, and to ultimately improve patient monitoring and individualize care, we conducted this systematic review and meta-analysis to determine the relationships between peripheral CRP, WBC, and the occurrence of DCI and poor functional outcome following aSAH.

\section{Materials and methods}

The present systematic review and meta-analysis was conducted in accordance with the Preferred Reporting Items for Systematic Reviews and Meta-Analyses (PRISMA) guidelines. Pubmed, EMBASE, and CENTRAL databases were searched until November 30, 2019 using the following keywords and word combinations to maximize search results:

((()(C-reactive protein [MeSH Terms]) OR white blood cell $[\mathrm{MeSH}$ Terms]) OR ((biomarkers [MeSH Terms]) OR predictor)) OR inflammation [MeSH Terms])) AND subarachnoid hemorrhage [MeSH Terms]

The search filters applied were: Abstract available; Humans. Reference lists of relevant studies were also handsearched for additional eligible studies.

\section{Selection criteria and data extraction}

Prospective and retrospective studies were selected that met the following inclusion criteria: (1) adults age $>18$ years with spontaneous SAH due to ruptured aneurysm, diagnosed by computed tomography angiogram (CTA) or digital subtraction angiography (DSA); (2) reporting adjusted odds ratios (controlling for baseline characteristics such as age, sex, clinical grades of severity at admission, and/or other known predictors) for the associations between the occurrence of delayed cerebral ischemia (DCI) or poor functional outcome and blood CRP/WBC collected either on admission or at the early phase of admission as part of the routine clinical workup. Studies of patients with traumatic SAH or SAH due to other secondary causes were excluded; those focused only on results of cerebrospinal fluid (CSF) CRP or WBC and did not provide data of peripheral CRP/WBC, non-human studies, letters, commentaries, reviews, editorials, case reports, proceedings and personal communications; and studies with no quantitative outcomes of interest were excluded. Studies were identified by the above search strategy by two independent reviewers (XM and FL). Where uncertainty existed regarding eligibility, a third reviewer (YZ) was consulted.

\section{Main outcome measures}

Outcome measures included the occurrence of DCI and poor functional outcome. DCI was defined as a new onset of focal neurological deficit or a deterioration of consciousness (delayed ischemic neurologic deficit, DIND); and/or a new infarct on CT or magnetic resonance (MR) imaging that was not visible initially and was attributed to cerebral vasospasm (delayed cerebral infraction). Functional outcomes were graded by modified Rankin Scale (mRS) or Glasgow outcomes scale (GOS). Poor functional outcomes were defined as mRS 3 or 4 to 6 , or GOS 1-3 from discharge to 1 -year follow-up.

The following data were extracted from each included study: author, year of publication, study design, patient number, sex and mean age of subjects, time from ictus to admission, time from ictus to sampling, procedures performed, clinical severity (i.e., Hunt and Hess scale; WFNS scale) and radiological severity (i.e., Fisher grade) on admission, criteria used for poor outcome, exclusion criteria, time point of outcome evaluation, and factors adjusted in the associations analyzed. Of note, if multiple 
analyses on CRP/WBC collected at different time point were reported in the same study, the earliest timeframe was selected for the meta-analysis (Fig. 1).

\section{Quality assessment}

We assessed the quality of included studies using the Newcastle-Ottawa scale (NOS) for cohort studies as recommended by the Cochrane Non-Randomized Studies Methods Working Group [17]. This scale awards a maximum of nine stars to each study: four stars for the adequate selection of cohort participants, two stars for comparability of cohort participants on the basis of the design and analysis, and three stars for the adequate ascertainment of outcomes. Quality assessment was performed by two independent reviewers (XM and FL), and a third reviewer (YZ) was consulted if any uncertainties occurred.

\section{Statistical analysis}

To investigate associations of early increase in CRP and WBC with the occurrence of DCI and poor functional outcome following aSAH, odds ratios (OR) with $95 \%$ confidence intervals (CI) were extracted from the included studies. An $\chi^{2}$-based test of homogeneity was performed using Cochran's $Q$ statistic and $I^{2} . I^{2}$ illustrates the percentage of total variability in effect estimates among trials that is due to heterogeneity rather than chance. A rough guide to interpretation of $I^{2}$ in the context of meta-analyses is as follows: (1) 0-40\%: may not be important; (2) 30-60\%: may represent moderate heterogeneity; (3) 50-90\%: may represent substantial heterogeneity; (4) 75-100\%: considerable heterogeneity [18]. In addition, a random-effects model was applied. Pooled effects were calculated and a two-sided $P$ value $<0.05$ was established as statistical significance. Sensitivity analysis was carried out using the leave-one-out approach. Publication bias analysis was not

Fig. 1 PRISMA flow diagram

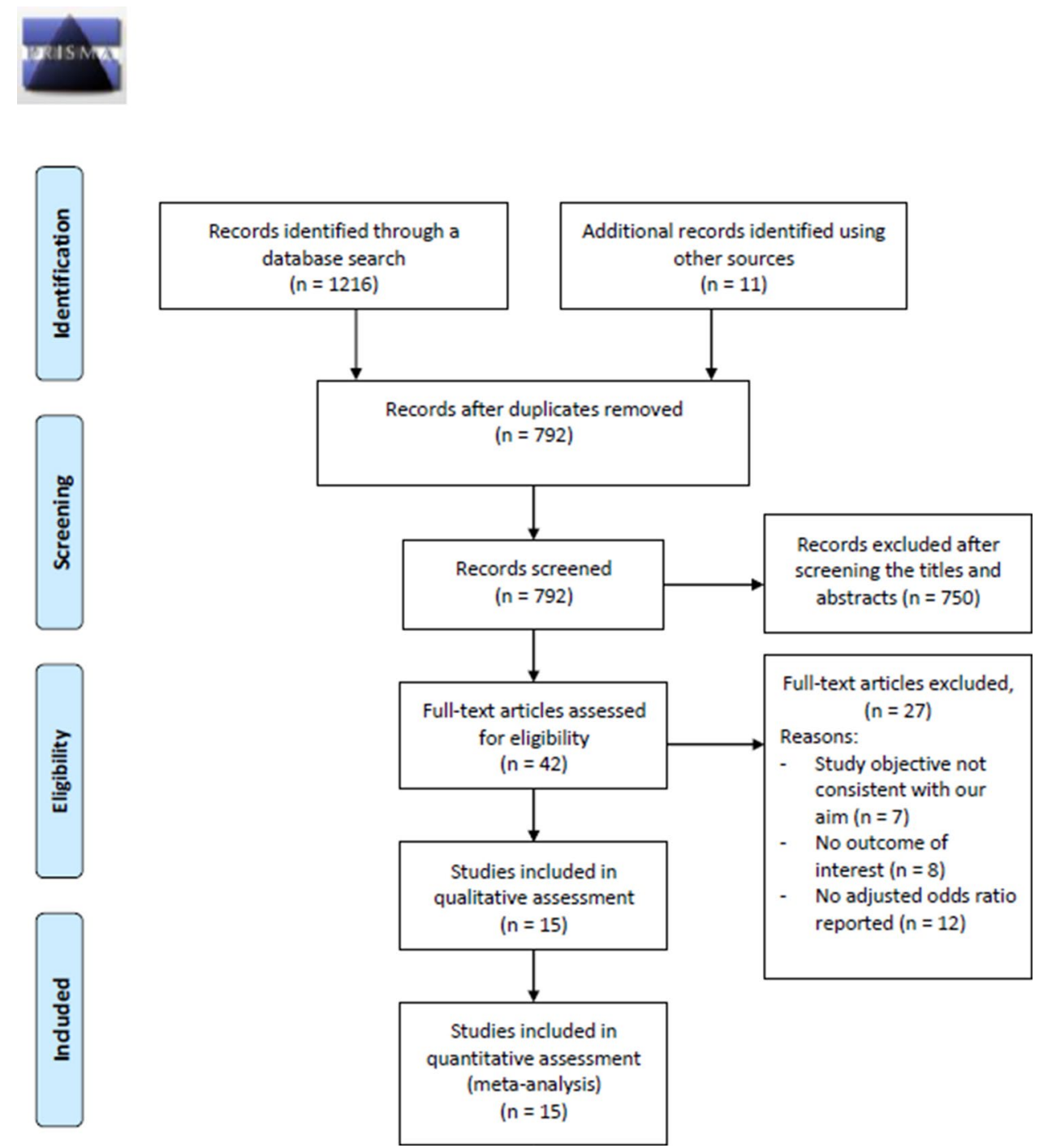


evaluated, because the number of studies was less than ten studies, too few to detect an asymmetric funnel [19]. All analyses were performed using Comprehensive MetaAnalysis statistical software, version 2.0 (Biostat, Englewood, NJ, USA).

\section{Results}

Among 42 eligible studies that underwent full-text review, 27 were excluded and 15 (5 retrospective, 10 prospective) that met the eligibility criteria were included [13-16, 20-30]. Characteristics of the included studies are summarized in Table 1. Overall, the 15 eligible studies analyzed the data of 3268 patients with aSAH. Patients' median age was 53 years (range 48-57 years) and the percentage of older adult males ranged from 24 to $56 \%$. Time from ictus to admission was within 24-96 h. Procedures performed, clinical and radiological severity are also summarized in Table 1 . Parameters studied, timeframe on sampling, outcome definitions, exclusion criteria and factors adjusted among the included studies are summarized in Table 2. Among the studies included, 11 studies assessed the parameters of interest by initial blood sampling at presentation. 2 studies were based on blood samples collected on postoperative days $1-2$, and 2 studies used blood samples collected during the first 14 days of admission. Clinical grade at presentation and patients' age were the most frequently adjusted factors (for clinical grade: 11 studies; for age: 8 studies) (Tables 1,2).

\section{Meta-analyses}

\section{Delayed cerebral ischemia}

Five studies [13, 16, 20, 23, 24] provided adjusted ORs and were included in the meta-analysis to determine associations between early increase in CRP and the occurrence of DCI following aSAH. Substantial heterogeneity was found across the five studies $\left(Q\right.$ statistic $=11.428, I^{2}=64.997 \%$, $P=0.022$ ). Early increase in CRP was significantly associated with higher risk of the occurrence of DCI (pooled OR $1.30,95 \%$ CI 1.10-1.54; $P=0.002$ ) (Fig. 2a).

Only four studies $[16,24,26,27]$ provided enough data of peripheral WBC and were included in the meta-analysis to determine the associations between early elevated WBC and occurrence of DCI. Substantial heterogeneity was found among the four studies $\left(Q\right.$ statistic $=13.691, I^{2}=78.089 \%$, $P=0.003$ ). Meta-analysis revealed there was no significant associations between increase in WBC and occurrence of DCI (pooled OR 1.13, 95\% CI $0.95-1.34 ; P=0.179$ ) (Fig. 2b).

\section{Poor functional outcomes}

The criteria used for defining poor functional outcomes are summarized in Table 2. Nine out of 15 studies were included in the meta-analysis to determine associations between increase in peripheral CRP and poor functional outcomes after aSAH. Early increase in CRP was not significantly associated with poor functional outcome (pooled OR 1.02, 95\% CI 1.00-1.04, $P=0.052$; heterogeneity: $Q$ statistic $\left.=24.571, I^{2}=71.511 \%, P=0.001\right)$ (Fig. 3a).

Results of meta-analysis of seven studies showed a significant association between early increase in WBC and increased risk of poor outcome (pooled OR 1.17, 95\% CI $1.07-1.28, P=0.001)$ with moderate heterogeneity among studies (heterogeneity: $Q$ statistic $=13.864, I^{2}=56.724 \%$, $P=0.031$ ) (Fig. 3b).

\section{Sensitivity analysis and publication bias}

Sensitivity analyses were performed using the leave-one-out approach in which the meta-analysis is performed with each study removed in turn (Table 3 ). The direction of combined estimates did not vary markedly with the removal of the studies, indicating that the meta-analysis was robust and the data were not overly influenced by any single study, only except for Sokół et al. [15] and Turner et al. [22] for CRP level associated with poor outcomes. After removing Sokół et al. [15] and Turner et al. [22], the results changed to significant.

Because of the small number of studies, the power of the tests for publication bias were too low to distinguish chance from real asymmetry.

\section{Quality assessment}

The result of quality assessment of included studies is shown in Supplementary Table 1. The total score ranged from 6 to 9 and the average score was 8 , suggesting that the studies included were of good quality (Supplementary Table 1).

\section{Discussion}

In the present review and meta-analysis, we assessed the associations between DCI/poor functional outcome following aSAH and blood CRP/WBC. These two parameters were selected for meta-analysis, because they were the most consistently assessed inflammatory markers in relation to aSAH prognosis in the literature. The results show increase in baseline CRP was significantly associated with increased risk of DCI following aSAH, but not with 
Table 1 Characteristics of studies included in this review and meta-analysis

\begin{tabular}{|c|c|c|c|c|c|c|c|c|c|}
\hline \multirow[t]{2}{*}{$\begin{array}{l}\text { First author } \\
\text { (year) }\end{array}$} & \multirow[t]{2}{*}{ Study design } & \multirow[t]{2}{*}{ No. of patients } & \multirow[t]{2}{*}{ Age (years) } & \multirow[t]{2}{*}{ Male (\%) } & \multirow[t]{2}{*}{$\begin{array}{l}\text { Procedure per- } \\
\text { formed }(\%)\end{array}$} & \multicolumn{2}{|c|}{$\begin{array}{l}\text { Clinical severity on admis- } \\
\text { sion }\end{array}$} & \multirow{2}{*}{$\begin{array}{l}\text { Radiological } \\
\text { severity on } \\
\text { admission }^{\mathrm{a}}\end{array}$} & \multirow{2}{*}{$\begin{array}{l}\text { Time from } \\
\text { ictus to admis- } \\
\text { sion }\end{array}$} \\
\hline & & & & & & Hunt and Hess & WFNS & & \\
\hline $\begin{array}{l}\text { Al-Mufti } \\
\text { (2019) }\end{array}$ & Prospective & 849 & $>53: 55 \%$ & 27 & Clipping: $69 \%$ & & $3-5: 47 \%$ & & $<24 \mathrm{~h}$ \\
\hline Wu (2019) & Retrospective & 122 & 55.3 & 39 & NA & 4-5: $19.7 \%$ & & $3-4: 89.3 \%$ & $<72 \mathrm{~h}$ \\
\hline Sokół (2018) & Prospective & 116 & 54.3 & 45 & Coiling: $100 \%$ & $\begin{array}{l}1-3: 52.5 \% \\
4-5: 47.5 \%\end{array}$ & $4 \mathrm{~b}$ & & $<24 \mathrm{~h}$ \\
\hline Frontera (2017) & Prospective & 50 & $55^{\mathrm{b}}$ & 41 & $\begin{array}{l}\text { None: } 25 \% \\
\text { Clipping: } 15 \% \\
\text { Coiling: } 56 \% \\
\text { Both: } 1 \%\end{array}$ & $\begin{array}{l}1-3: 77 \% \\
4-5: 23 \%\end{array}$ & & & $<72 \mathrm{~h}$ \\
\hline Siegler (2017) & Retrospective & 179 & $54^{\mathrm{b}}$ & 24 & $\begin{array}{l}\text { Clipping: } \\
26.8 \% \\
\text { Coiling: } 67 \% \\
\text { Other/None: } \\
6.1 \%\end{array}$ & $4-5: 25 \%$ & & $3-4: 80 \%$ & NA \\
\hline Tao (2017) & Prospective & 247 & 55.9 & 36 & $\begin{array}{l}\text { Clipping: } \\
80.2 \% \\
\text { Coiling: } 19.8 \%\end{array}$ & & $2^{\mathrm{b}}$ & $3^{\mathrm{b}}$ & $<24 \mathrm{~h}$ \\
\hline Chugh (2015) & Retrospective & 40 & 52.8 & 25 & $\begin{array}{l}\text { Clipping: } 30 \% \\
\text { Coiling: } 63 \%\end{array}$ & $4-5: 30 \%$ & & 3: $100 \%$ & $<24 \mathrm{~h}$ \\
\hline Csajbok (2015) & Prospective & 98 & $57^{\mathrm{b}}$ & 24 & Coiling: $100 \%$ & & $\begin{array}{l}1: 34.7 \% \\
2: 30.6 \% \\
3: 3.1 \% \\
4: 20.4 \% \\
5: 11.2 \%\end{array}$ & $\begin{array}{l}2: 3.1 \% \\
3: 31.6 \% \\
4: 65.3 \%\end{array}$ & $<24 \mathrm{~h}$ \\
\hline Turner (2015) & Prospective & 803 & 50 & 31 & NA & & $\begin{array}{l}1: 49 \% \\
2: 25 \% \\
3: 4 \% \\
4: 13 \% \\
5: 10 \%\end{array}$ & $\begin{array}{l}1: 2 \% \\
2: 14 \% \\
3: 33 \% \\
4: 51 \%\end{array}$ & $<96 \mathrm{~h}$ \\
\hline Romero (2014) & Prospective & 100 & 48 & 56 & $\begin{array}{l}\text { Clipping: } 57 \% \\
\text { Coiling: } 43 \%\end{array}$ & & 2.5 & 1.5 & $<24 \mathrm{~h}$ \\
\hline $\begin{array}{l}\text { McMahon } \\
\text { (2013) }\end{array}$ & Prospective & 149 & $50^{\mathrm{b}}$ & 33 & $\begin{array}{l}\text { Clipping: } 10 \% \\
\text { Coiling: } 86 \% \\
\text { Conservative: } \\
3 \%\end{array}$ & & $\begin{array}{l}1: 67 \% \\
2: 21 \% \\
3: 6 \% \\
4: 3 \% \\
5: 3 \%\end{array}$ & $\begin{array}{l}1: 5 \% \\
2: 26 \% \\
3: 52 \% \\
4: 18 \%\end{array}$ & NA \\
\hline Muroi (2013) & Prospective & 138 & 54.4 & 34 & $\begin{array}{l}\text { Clipping: } 57 \% \\
\text { Coiling: } 43 \%\end{array}$ & & $\begin{array}{l}1-3: 66 \% \\
4-5: 34 \%\end{array}$ & $\begin{array}{l}1-2: 16 \% \\
3: 61 \% \\
4: 23 \%\end{array}$ & $<36 \mathrm{~h}$ \\
\hline Jeon (2012) & Retrospective & 93 & NA & 35 & $\begin{array}{l}\text { Clipping: } \\
21.5 \% \\
\text { Coiling: } 78.5 \%\end{array}$ & $\begin{array}{l}1-2: 66.7 \% \\
3-4: 33.3 \%\end{array}$ & & $\begin{array}{l}2,4: 60.2 \% \\
3: 39.8 \%\end{array}$ & NA \\
\hline Juvela (2012) & Prospective & 178 & 50.3 & 49 & $\begin{array}{l}\text { Clipping: } 97 \% \\
\text { Coiling: } 3 \%\end{array}$ & & $\begin{array}{l}1: 54 \% \\
2-3: 25 \% \\
4-5: 21 \%\end{array}$ & $\begin{array}{l}1-2,4: 33 \% \\
3: 67 \%\end{array}$ & $<48 \mathrm{~h}$ \\
\hline Kasius (2009) & Retrospective & 106 & NA & 33 & $\begin{array}{l}\text { Clipping: } \\
\text { 71.7\% } \\
\text { Coiling: } 12.3 \%\end{array}$ & & $\begin{array}{l}1: 41.5 \% \\
2: 29.2 \% \\
3: 5.7 \% \\
4: 16.9 \% \\
5: 6.6 \%\end{array}$ & & $<72 \mathrm{~h}$ \\
\hline
\end{tabular}

WFNS World Federation of Neurosurgical Societies, NA not applicable

${ }^{\text {a}}$ Fisher grade

${ }^{\mathrm{b}}$ Data displayed as median

poor functional outcome at follow-up. Increase in baseline WBC was not associated with DCI, however, were significantly associated with poor functional outcome.
Prediction of the occurrence of DCI and poor outcome is of critical value for aSAH management. It is known that the need for special laboratory test or collection of CSF 


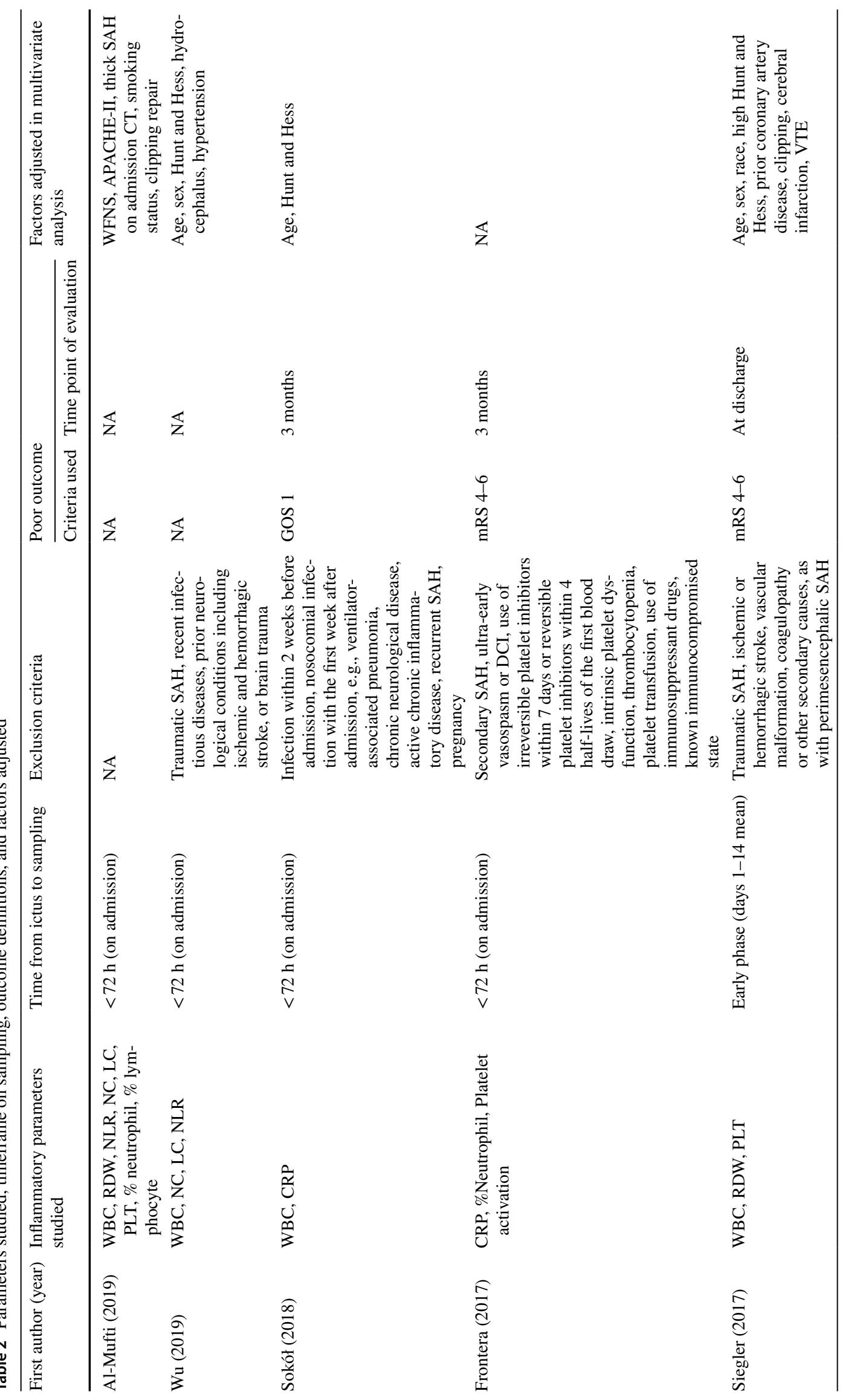




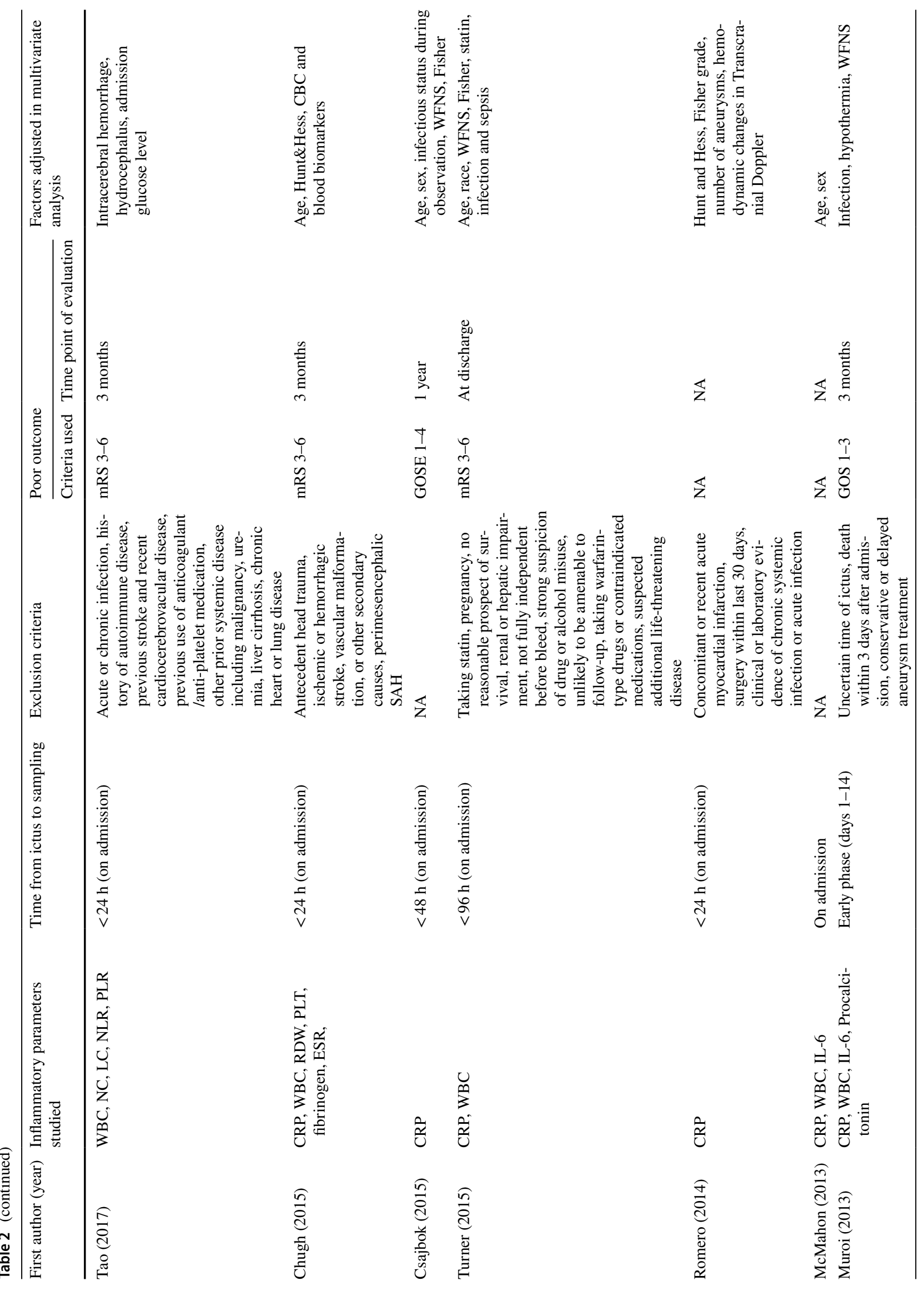




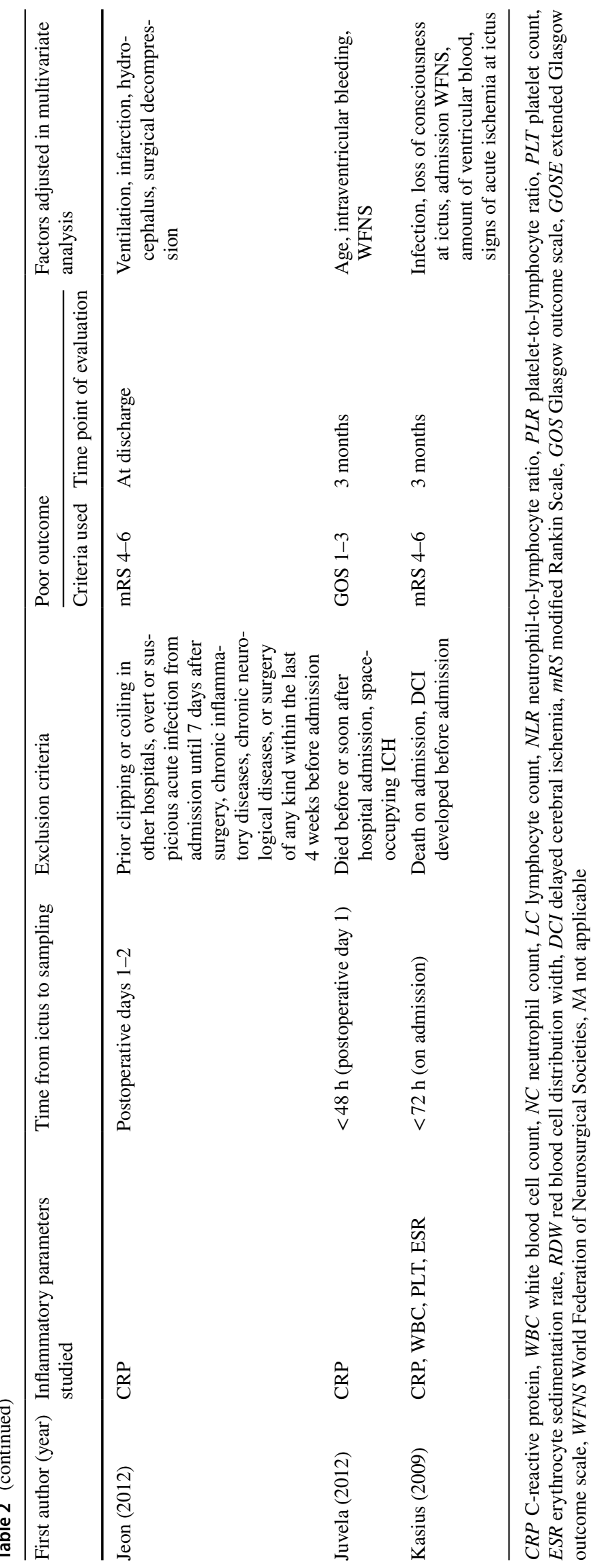


(A)

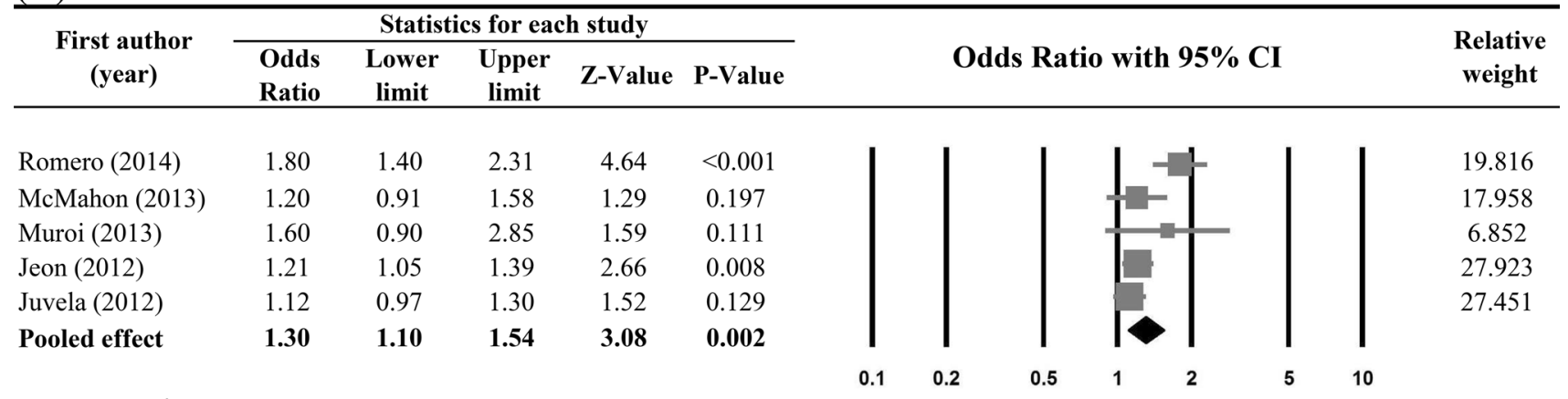

Heterogeneity test

$\mathrm{Q}=11.428, \mathrm{df}=4, \mathrm{P}=0.022 \mathrm{I}$-square $=64.997 \%$

(B)

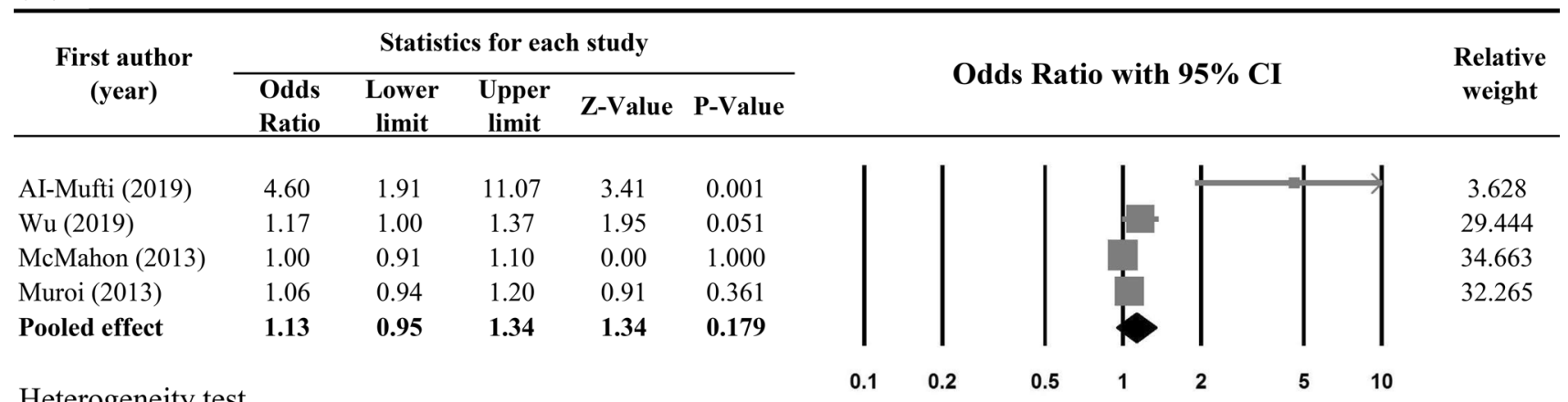

Heterogeneity test

$\mathrm{Q}=13.691, \mathrm{df}=3, \mathrm{P}=0.003 \mathrm{I}$-square $=78.089 \%$

Lower level of WBC Higher level of WBC

Fig. 2 Meta-analysis on the associations between occurrence of DCI and a CRP level and b WBC

sample limits the clinical utility of a biomarker, and this highlights the need for searching for a quick, sensitive and safe biomarker which is easy to obtain for evaluating prognosis in aSAH. In addition, there is increasing evidence on the role of inflammation in the pathogenesis of DCI and poor outcomes following aSAH [27]. Among the common readily available markers for systemic inflammation, many studies have investigated the independent role of early increase in CRP and WBC as risk factors for unfavorable outcomes after aSAH. However, the correlations remained inconclusive and there is no consensus on their value for assessing prognosis in aSAH. For example, early phase CRP after aSAH have predicted poor outcome and/ or DCI in a few studies [14, 20, 23], while not in others $[16,24]$. Conflict results were also observed with respect to studies on WBC $[15,26,30]$. On the other hand, there is an extensive body of literature identifying blood CRP and WBC as an independent and relatively strong predictive marker for outcomes after ischemic stroke [31-33], it is reasonable for expecting these associations in aSAH.

Suprisingly, this present meta-analysis found early increase in CRP is only associated with DCI but not later poor functional outcomes. There is a known association between DCI and worse clinical outcomes. However, sensitivity analysis showed that removing Sokół et al. [15] or Turner et al. [22] resulted in a significant association between poor outcome and CRP, suggesting the pooled estimate was overly impacted by these two studies. There was a relatively distinct definition of poor outcome (GOS 1) in Sokół et al. [15], and longer duration from hemorrhage onset to sampling in Turner et al. [22] than in other studies, which may help to explain the discrepancies.

Moderate to substantial heterogeneity was detected in the analyses of present review. A number of factors may be responsible for the heterogeneity. Firstly, the time point of outcome evaluation among the studies was not unified and 
(A)

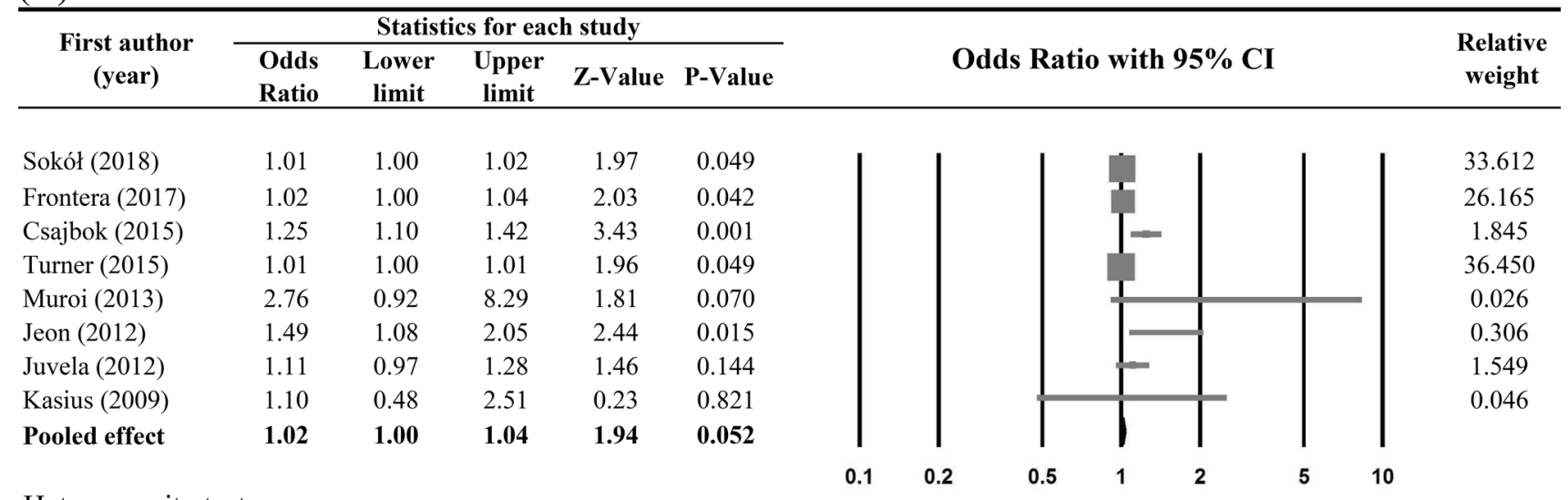

Heterogeneity test

$\mathrm{Q}=24.571, \mathrm{df}=7, \mathrm{P}=0.001$ I-square $=71.511 \%$

Lower level of CRP Higher level of CRP

(B)

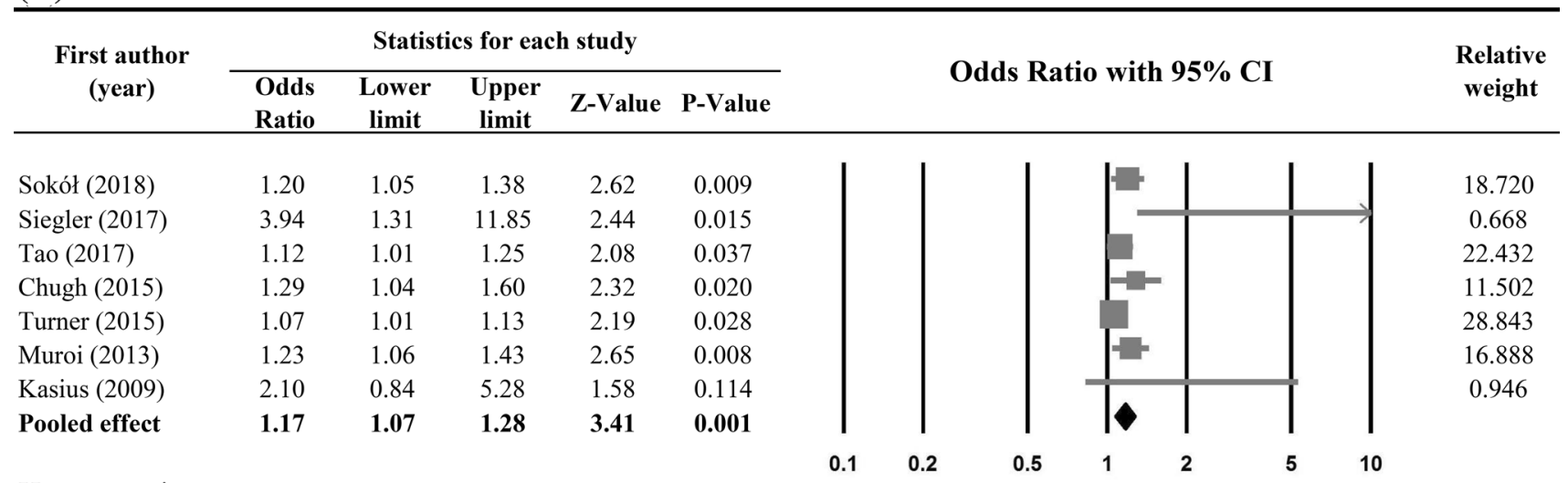

Heterogeneity test

$\mathrm{Q}=13.864, \mathrm{df}=6, \mathrm{P}=0.031$ I-square $=56.724 \%$

Lower level of WBC Higher level of WBC

Fig. 3 Meta-analysis on the associations between poor function outcome and a CRP level and $\mathbf{b}$ WBC

not always clearly stated. Secondly, the aim of the present review was to analyze the associative role of early CRP/ WBC. While most studies provided data of the first sampling on presentation, and although not markedly varied, there were still some variations in the timeframe of sample collection, and may contribute to the heterogeneity, since CRP is a sensitive marker and could have changed rapidly. Patient baseline characteristics, procedures performed, as well as factors adjusted for analyses differed across the studies, which may also contribute to the heterogeneity.

No meta-analyses have quantitively analyzed the associations between CRP/WBC and DCI/functional outcome following $\mathrm{SAH}$, although several recent systematic reviews have provided qualitative assessment of a broad range of potential biomarkers for aSAH outcomes, including CRP and WBC [26, 34-36]. Jabbarli et al. [34] identified 21 genetic and non-genetic laboratory DCI biomarkers as 'predictive biomarkers' with level II evidence; peripheral CRP and WBC were evaluated in that review, of which level of evidence was considered insufficient, thus were allocated as 'non-conclusive' markers. Hong et al. [35] showed that serum CRP had increased specificity and sensitivity as a marker of inflammation after $\mathrm{SAH}$ and, as such, was a useful predictive indicator of vasospasm and less favorable functional outcomes after SAH. Authors of another study suggested utilizing CRP as prognostic indicator of SAH has a special value in sedated patients, because clinical condition of these patients is difficult to estimate [13].

The mechanisms underlying the associations observed remain unclear. Al-Mufti et al. [26] argued that WBCs 
Table 3 Sensitivity analysis

\begin{tabular}{|c|c|c|c|c|c|}
\hline \multirow[t]{2}{*}{ First author (year) } & \multicolumn{5}{|c|}{ Statistics with study removed } \\
\hline & Points & Lower limit & Upper limit & $Z$ value & $P$ value \\
\hline \multicolumn{6}{|l|}{ CRP and DCI } \\
\hline Romero (2014) & 1.18 & 1.07 & 1.30 & 3.45 & 0.001 \\
\hline McMahon (2013) & 1.34 & 1.09 & 1.65 & 2.74 & 0.006 \\
\hline Muroi (2013) & 1.29 & 1.07 & 1.54 & 2.74 & 0.006 \\
\hline Jeon (2012) & 1.36 & 1.05 & 1.76 & 2.35 & 0.019 \\
\hline Juvela (2012) & 1.39 & 1.11 & 1.73 & 2.92 & 0.004 \\
\hline \multicolumn{6}{|l|}{ WBC and DCI } \\
\hline AI-Mufti (2019) & 1.06 & 0.97 & 1.15 & 1.26 & 0.207 \\
\hline Wu (2019) & 1.14 & 0.89 & 1.46 & 1.04 & 0.299 \\
\hline McMahon (2013) & 1.27 & 0.94 & 1.71 & 1.58 & 0.115 \\
\hline Muroi (2013) & 1.24 & 0.91 & 1.69 & 1.39 & 0.165 \\
\hline \multicolumn{6}{|c|}{$\mathrm{CRP}$ and poor outcome } \\
\hline Sokół (2018) & 1.04 & 1.00 & 1.08 & 2.08 & 0.038 \\
\hline Frontera (2017) & 1.02 & 1.00 & 1.04 & 1.61 & 0.108 \\
\hline Csajbok (2015) & 1.01 & 1.00 & 1.02 & 1.79 & 0.074 \\
\hline Turner (2015) & 1.05 & 1.00 & 1.09 & 2.20 & 0.028 \\
\hline Muroi (2013) & 1.02 & 1.00 & 1.03 & 1.93 & 0.053 \\
\hline Jeon (2012) & 1.02 & 1.00 & 1.03 & 1.88 & 0.061 \\
\hline Juvela (2012) & 1.02 & 1.00 & 1.03 & 1.79 & 0.074 \\
\hline Kasius (2009) & 1.02 & 1.00 & 1.04 & 1.93 & 0.054 \\
\hline \multicolumn{6}{|c|}{ WBC and poor outcome } \\
\hline Sokół (2018) & 1.17 & 1.05 & 1.31 & 2.85 & 0.004 \\
\hline Siegler (2017) & 1.15 & 1.07 & 1.24 & 3.70 & 0.000 \\
\hline Tao (2017) & 1.20 & 1.06 & 1.37 & 2.91 & 0.004 \\
\hline Chugh (2015) & 1.16 & 1.05 & 1.27 & 2.96 & 0.003 \\
\hline Turner (2015) & 1.21 & 1.10 & 1.34 & 3.73 & 0.000 \\
\hline Muroi (2013) & 1.16 & 1.05 & 1.29 & 2.86 & 0.004 \\
\hline Kasius (2009) & 1.16 & 1.06 & 1.27 & 3.35 & 0.001 \\
\hline
\end{tabular}

produce reactive oxygen species (ROS) and disrupt the blood-brain barrier during extravasation into the cerebrum, which may contribute to DCI risk and poor outcomes in SAH. Those authors supported this by citing the finding of lymphocytic infiltrates on histopathologic staining of resected aneurysm tissue, which also have been considered as independent predictors of vasospasm [21, 30]. It is unlikely for us to infer whether or specifically how CRP/ WBC may participate in the pathogenesis of DCI or the causal process in poor function after SAH. The observed associations may be explained by, as a previous study pointed out, that systemic inflammation as indicated by these parameters increases susceptibility to the development of DCI after SAH [16].

Authors of the review cited above also cautioned that CRP is an acute phase sensitive but non-specific inflammatory marker and it is not produced by brain tissue. SAH commonly has a secondary process such as pneumonia or other infection, which may lower the potential prognostic value of CRP [35]. In the present meta-analysis, differences in patients' baseline characteristics and initial severity have been partially addressed by the multivariable models used in the included study adjusting for relevant factors (Table 2). Therefore, the pool estimation of CRP/WBC in related to outcome was not only a reflection of SAH severity. However, although several studies have, not all the included studies have excluded or adjusted for infection in their analyses. Also, assessment of infection is challenging and might have chance to be inaccurate [24]. Therefore, despite the fact we focused on the early period of aSAH and the parameters was mostly collected on admission in which infection was unlikely to present, the confounding by infection might have not been fully compensated. We are not able to make a further adjustment because of lacking raw data of individual studies.

Since CRP and WBC are relatively non-specific markers of inflammation. Evaluating other inflammatory markers such as Interleukin-6 (IL-6) and procalcitonin may provide additional insight. Unfortunately, few studies in the literature have looked into them; therefore, meta-analysis cannot 
be performed. Muroi et al. [24] investigated the relationships among IL-6, procalcitonin and outcomes after SAH, reporting a significant correlation between IL-6 and poor outcome (adjusted OR 4.03, $P=0.02$ ) and DCI (adjusted OR 2.78, $P=0.03$ ). Those authors concluded higher IL-6 levels might be a useful parameter to monitor. McMahon et al. [16] assessed the relation between IL-6 and DCI, while no significant association was found (adjusted odds ratio $1.1, P=0.46)$. Another novel biomarker, red cell distribution width (RDW) which is strongly linked to inflammatory and thrombotic disease, was evaluated by three of the included studies [26, 29, 30]. Chugh et al. [30] reported a significant association between maximum daily RDW during first 10 days following hemorrhage onset and poor clinical outcome (adjusted OR 2.3, $P=0.014$ ). Siegler et al. [29] also reported a significant association between elevated 14-day mean RDW and poor functional outcome at discharge (mRS $>4$, adjusted OR 2.59, $P=0.040$ ). However, elevated RDW in first $72 \mathrm{~h}$ was not associated with poor outcome in another study [26].

In the present review, we only identified associations and no predictive power was estimated due to insufficient reports. Among the studies included, Sokól et al. [15] reported the accuracy for prognosis of area under the curve (AUC) 0.74 $(P<0.05)$ in early CRP and poor outcome by calculating receiver operating characteristic (ROC) curve. Turner et al. [22] reported an AUC 0.779 in early CRP for predicting poor outcome at discharge among good grade patients. AlMufti et al. [26] reported combined utilizing Fisher grade and elevated admission WBC together had an AUC over 0.7, which was more accurate than Fisher grade alone. These reports together highlighted the potential predicting power of early CRP/WBC on aSAH outcomes, which is valuable to be confirmed in the future studies.

\section{Strengths and limitations}

This is the first meta-analysis to evaluate the role of peripheral CRP and WBC in outcomes of aSAH. The studies included were determined to be of good quality. This review has several limitations that should be considered when interpreting the results. Although fifteen studies were included, the number of studies included in the analyses for individual outcome were relatively small, which limited the statistical power. Initial severity of ictus, comorbidities, infectious status, surgery and external ventricular drain may largely influence the parameters studied, and were not fully compensated in the analyses. Another weakness of this meta-analysis is that only association was identified, no predictive power was estimated due to the lack of consistent reports. Inflammatory markers other than CRP/WBC, as well as different subtypes of WBCs were not analyzed due to insufficient data. Despite the described limitations, the present review give insight into the relationships of systemic inflammation with DCI and outcome after aSAH in general.

\section{Conclusions}

There appears to be some evidence indicating early increase in peripheral CRP is independently associated with the occurrence of DCI after SAH, and WBC associated with poor functional outcome at follow-up. Strong conclusion cannot be made due to the small study number, betweenstudy heterogeneity and suspicion of uncontrolled factors. Future well-designed studies with prognostic accuracy evaluated, factors precisely adjusted and inflammatory biomarkers other than CRP/WBC assessed are highly warranted to determine whether monitoring circulating markers of inflammation benefits the risk identification after aSAH.

Funding No funding was received for this research.

\section{Compliance with ethical standards}

Conflict of Interest All authors certify that they have no affiliations with or involvement in any organization or entity with any financial interest (such as honoraria; educational grants; participation in speakers' bureaus; membership, employment, consultancies, stock ownership, or other equity interest; and expert testimony or patent-licensing arrangements), or non-financial interest (such as personal or professional relationships, affiliations, knowledge or beliefs) in the subject matter or materials discussed in this manuscript.

Ethical approval We exclusively assessed previously published data. Thus, ethical approval was not required for the conduction of the metaanalysis and systematic review.

\section{References}

1. De Rooij NK, Linn FHH, van der Plas JA, Algra A, Rinkel GJE (2007) Incidence of subarachnoid haemorrhage: a systematic review with emphasis on region, age, gender and time trends. J Neurol Neurosurg Psychiatry 78(12):1365-1372

2. Rinkel GJ, Algra A (2011) Long-term outcomes of patients with aneurysmal subarachnoid haemorrhage. Lancet Neurol 10(4):349-356

3. Macdonald RL, Schweizer TA (2017) Spontaneous subarachnoid haemorrhage. Lancet 389(10069):655-666. https://doi. org/10.1016/s0140-6736(16)30668-7

4. Boluijt J, Meijers JC, Rinkel GJ, Vergouwen MD (2015) Hemostasis and fibrinolysis in delayed cerebral ischemia after aneurysmal subarachnoid hemorrhage: a systematic review. J Cereb Blood Flow Metab 35(5):724-733

5. Francoeur CL, Mayer SA (2016) Management of delayed cerebral ischemia after subarachnoid hemorrhage. Crit Care 20(1):277-281

6. Rabinstein AA, Weigand S, Atkinson JL, Wijdicks EF (2005) Patterns of cerebral infarction in aneurysmal subarachnoid hemorrhage. Stroke 36(5):992-997 
7. Dankbaar JW, Rijsdijk M, Schaaf ICvd, Velthuis BK, Wermer MJH, Rinkel GJE, (2009) Relationship between vasospasm, cerebral perfusion, and delayed cerebral ischemia after aneurysmal subarachnoid hemorrhage. Neuroradiology 51(12):813-819

8. Vergouwen MDI, Ilodigwe D, Macdonald RL (2011) Cerebral infarction after subarachnoid hemorrhage contributes to poor outcome by vasospasm-dependent and -independent effects. Stroke 42(4):924-929

9. Al-Mufti F, Amuluru K, Smith B, Damodara N, El-Ghanem M, Singh IP, Dangayach N, Gandhi CD (2017) Early emerging markers of delayed cerebral ischemia following aneurysmal subarachnoid hemorrhage: a literature review. World Neurosurg 107:148-159. https://doi.org/10.1016/j.wneu.2017.07.114

10. Galea JP, Dulhanty L, Patel HC (2017) Predictors of outcome in aneurysmal subarachnoid hemorrhage patients. Stroke 48(11):2958-2963. https://doi.org/10.1161/strokeaha.117.01777 7

11. Matano F, Fujiki Y, Mizunari T, Koketsu K, Tamaki T, Murai Y, Yokota H, Morita A (2019) Serum glucose and potassium ratio as risk factors for cerebral vasospasm after aneurysmal subarachnoid hemorrhage. J Stroke Cerebrovasc Dis 28(7):1951-1957. https:// doi.org/10.1016/j.jstrokecerebrovasdis.2019.03.041

12. Sadamasa N, Yoshida K, Narumi O, Chin M, Yamagata S (2011) Prediction of mortality by hematological parameters on admission in patients with subarachnoid hemorrhage. Neurol Med Chir 51(11):745-748

13. Juvela S, Kuhmonen J, Siironen J (2012) C-reactive protein as predictor for poor outcome after aneurysmal subarachnoid haemorrhage. Acta Neurochir 154(3):397-404. https://doi.org/10.1007/ s00701-011-1243-7

14. Csajbok LZ, Nylén K, Öst M, Sonander H, Nellgård B (2015) In-hospital C-reactive protein predicts outcome after aneurysmal subarachnoid haemorrhage treated by endovascular coiling. Acta Anaesthesiol Scand 59(2):255-264

15. Sokół B, Wąsik N, Więckowska B, Mańko W, Juszkat R, Jankowski R (2018) Predicting mortality in subarachnoid haemorrhage based on first-week routine blood tests. J Clin Neurosci 58:100-107. https://doi.org/10.1016/j.jocn.2018.09.016

16. McMahon CJ, Hopkins S, Vail A, King AT, Smith D, Illingworth KJ, Clark S, Rothwell NJ, Tyrrell PJ (2013) Inflammation as a predictor for delayed cerebral ischemia after aneurysmal subarachnoid haemorrhage. J Neurointerv Surg 5(6):512-517. https ://doi.org/10.1136/neurintsurg-2012-010386

17. Stang A (2010) Critical evaluation of the Newcastle-Ottawa scale for the assessment of the quality of nonrandomized studies in meta-analyses. Eur J Epidemiol 25(9):603-605. https://doi. org/10.1007/s10654-010-9491-z

18. Higgins JPT, Thomas J, Chandler J, Cumpston M, Li T, Page MJ, Welch VA (2009) Cochrane handbook for systematic reviews of interventions. 2nd edn. Chichester (UK): John Wiley \& Sons

19. Sterne JAC, Sutton AJ, Ioannidis JPA, Terrin N, Jones DR, Lau J, Carpenter J, Rucker G, Harbord RM, Schmid CH, Tetzlaff J, Deeks JJ, Peters J, Macaskill P, Schwarzer G, Duval S, Altman DG, Moher D, Higgins JPT (2011) Recommendations for examining and interpreting funnel plot asymmetry in meta-analyses of randomised controlled trials. BMJ 343(1):d4002-d4002. https:// doi.org/10.1136/bmj.d4002

20. Romero FR, Cataneo DC, Cataneo AJ (2014) C-reactive protein and vasospasm after aneurysmal subarachnoid hemorrhage. Acta Cirurgica Brasileira 29(5):340-345. https://doi.org/10.1590/s0102 $-86502014000500009$

21. Frontera JA, Provencio JJ, Sehba FA, McIntyre TM, Nowacki AS, Gordon E, Weimer JM, Aledort L (2017) The role of platelet activation and inflammation in early brain injury following subarachnoid hemorrhage. Neurocrit Care 26(1):48-57. https:// doi.org/10.1007/s12028-016-0292-4
22. Turner CL, Budohoski K, Smith C, Hutchinson PJ, Kirkpatrick PJ, Murray GD, Collaborators S (2015) Elevated baseline C-reactive protein as a predictor of outcome after aneurysmal subarachnoid hemorrhage: data from the simvastatin in aneurysmal subarachnoid hemorrhage (STASH) trial. Neurosurgery 77(5):786-792. https://doi.org/10.1227/NEU.0000000000000963(discussion 792-783)

23. Jeon Y-T, Lee J-H, Lee H, Lee H-K, Hwang J-W, Lim Y-J, Park H-P (2012) The postoperative C-reactive protein level can be a useful prognostic factor for poor outcome and symptomatic vasospasm in patients with aneurysmal subarachnoid hemorrhage. $\mathrm{J}$ Neurosurg Anesthesiol 24(4):317-324

24. Muroi C, Hugelshofer M, Seule M, Tastan I, Fujioka M, Mishima K, Keller E (2013) Correlation among systemic inflammatory parameter, occurrence of delayed neurological deficits, and outcome after aneurysmal subarachnoid hemorrhage. Neurosurgery 72(3):367-375. https://doi.org/10.1227/NEU.0b013e31828048ce

25. Kasius KM, Frijns CJM, Algra A, Rinkel GJE (2010) Association of platelet and leukocyte counts with delayed cerebral ischemia in aneurysmal subarachnoid hemorrhage. Cerebrovasc Dis 29(6):576-583

26. Al-Mufti F, Misiolek KA, Roh D, Alawi A, Bauerschmidt A, Park S, Agarwal S, Meyers PM, Connolly ES, Claassen J (2019) White blood cell count improves prediction of delayed cerebral ischemia following aneurysmal subarachnoid hemorrhage. Neurosurgery 84(2):397-403

27. Wu Y, He Q, Wei Y, Zhu J, He Z, Zhang X, Guo Z, Xu R, Cheng C, Huang Z, Sun X (2019) The association of neutrophil-to-lymphocyte ratio and delayed cerebral ischemia in patients with aneurysmal subarachnoid hemorrhage: possible involvement of cerebral blood perfusion. Neuropsychiatr Dis Treat 15:1001-1007. https://doi.org/10.2147/ndt.S190477

28. Tao C, Wang J, Hu X, Ma J, Li H, You C (2017) Clinical value of neutrophil to lymphocyte and platelet to lymphocyte ratio after aneurysmal subarachnoid hemorrhage. Neurocrit Care 26(3):393401. https://doi.org/10.1007/s12028-016-0332-0

29. Siegler JE, Marcaccio C, Nawalinski K, Quattrone F, Sandsmark DK, Maloney-Wilensky E, Frangos S, Levine JM, Stein SC, Kasner SE, Kumar MA (2016) Elevated red cell distribution width is associated with cerebral infarction in aneurysmal subarachnoid hemorrhage. Neurocrit Care 26(1):26-33. https://doi.org/10.1007/ s12028-016-0306-2

30. Chugh C, Nyirjesy SC, Nawalinski KP, Sandsmark DK, Frangos S, Maloney-Wilensky E, Stein SC, Levine JM, Kasner SE, Kumar MA (2015) Red blood cell distribution width is associated with poor clinical outcome after subarachnoid hemorrhage: a pilot study. Neurocrit Care 23(2):217-224. https://doi.org/10.1007/ s12028-015-0117-x

31. Yu B, Yang P, Xu X, Shao L (2019) C-reactive protein for predicting all-cause mortality in patients with acute ischemic stroke: a meta-analysis. Biosci Rep. https://doi.org/10.1042/bsr20181135

32. Geng H-H, Wang X-W, Fu R-L, Jing M-J, Huang L-L, Zhang Q, Wang X-X, Wang P-X (2016) The relationship between C-reactive protein level and discharge outcome in patients with acute ischemic stroke. Int J Environ Res Public Health. https://doi. org/10.3390/ijerph13070636

33. Turnbull FM, Whiteley W, Jackson C, Lewis S, Lowe G, Rumley A, Sandercock P, Wardlaw J, Dennis M, Sudlow C (2009) Inflammatory markers and poor outcome after stroke: a prospective cohort study and systematic review of interleukin-6. PLoS Med. https://doi.org/10.1371/journal.pmed.1000145

34. Jabbarli R, Pierscianek D, Darkwah Oppong M, Sato T, Dammann P, Wrede KH, Kaier K, Köhrmann M, Forsting M, Kleinschnitz C, Roos A, Sure U (2018) Laboratory biomarkers of delayed cerebral ischemia after subarachnoid hemorrhage: a systematic review. Neurosurg Rev. https://doi.org/10.1007/s10143-018-1037-y 
35. Hong CM, Tosun C, Kurland DB, Gerzanich V, Schreibman D, Simard JM (2014) Biomarkers as outcome predictors in subarachnoid hemorrhage—a systematic review. Biomarkers 19(2):95108. https://doi.org/10.3109/1354750x.2014.881418

36. de Rooij NK, Rinkel GJE, Dankbaar JW, Frijns CJM (2013) Delayed cerebral ischemia after subarachnoid hemorrhage. Stroke 44(1):43-54. https://doi.org/10.1161/strokeaha.112.674291
Publisher's Note Springer Nature remains neutral with regard to jurisdictional claims in published maps and institutional affiliations. 